\title{
Multiple disseminated keratoacanthoma-like nodules: a rare form of distant metastases to the skin
}

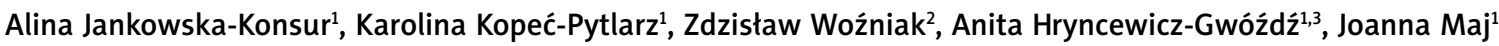

1Department of Dermatology, Venereology and Allergology, Wroclaw Medical University, Wroclaw, Poland

2Department of Pathomorphology, Wroclaw Medical University, Wroclaw, Poland

${ }^{3}$ Clinic of Dermatology, Venerology and Allergology, Faculty of Medicine and Dentistry, Wroclaw Medical University, Wroclaw, Poland

Adv Dermatol Allergol 2018; XXXV (5): 535-537

DOI: https://doi.org/10.5114/ada.2018.77245

Cutaneous metastases are found in approximately $0.7-10.4 \%$ of internal malignancies and they may rarely be the first symptom of the underlying neoplasm [1]. Typically, cutaneous secondaries present as a single, erythematous nodule, occasionally ulcerated, however, other presentations, including erysipelas carcinomatosa, alopecia neoplastica or carcinoma en cuirasse in the course of the breast cancer, or angiomatous tumors in the course of renal carcinoma may be occasionally ob- served. The metastases assimilating keratoacanthomas are extremely rare $[2,3]$.

Herein, we present a 72-year-old man, cigarette smoker, who was referred to our department with disseminated skin tumors of unknown etiology. On admission, domeshaped, inflamed tumors, some of them with central, keratin-filled craters, clinically mimicking keratoacanthomas were observed on the scalp, forehead, nose, neck and trunk (back and left shoulder) (Figures 1, 2). All the
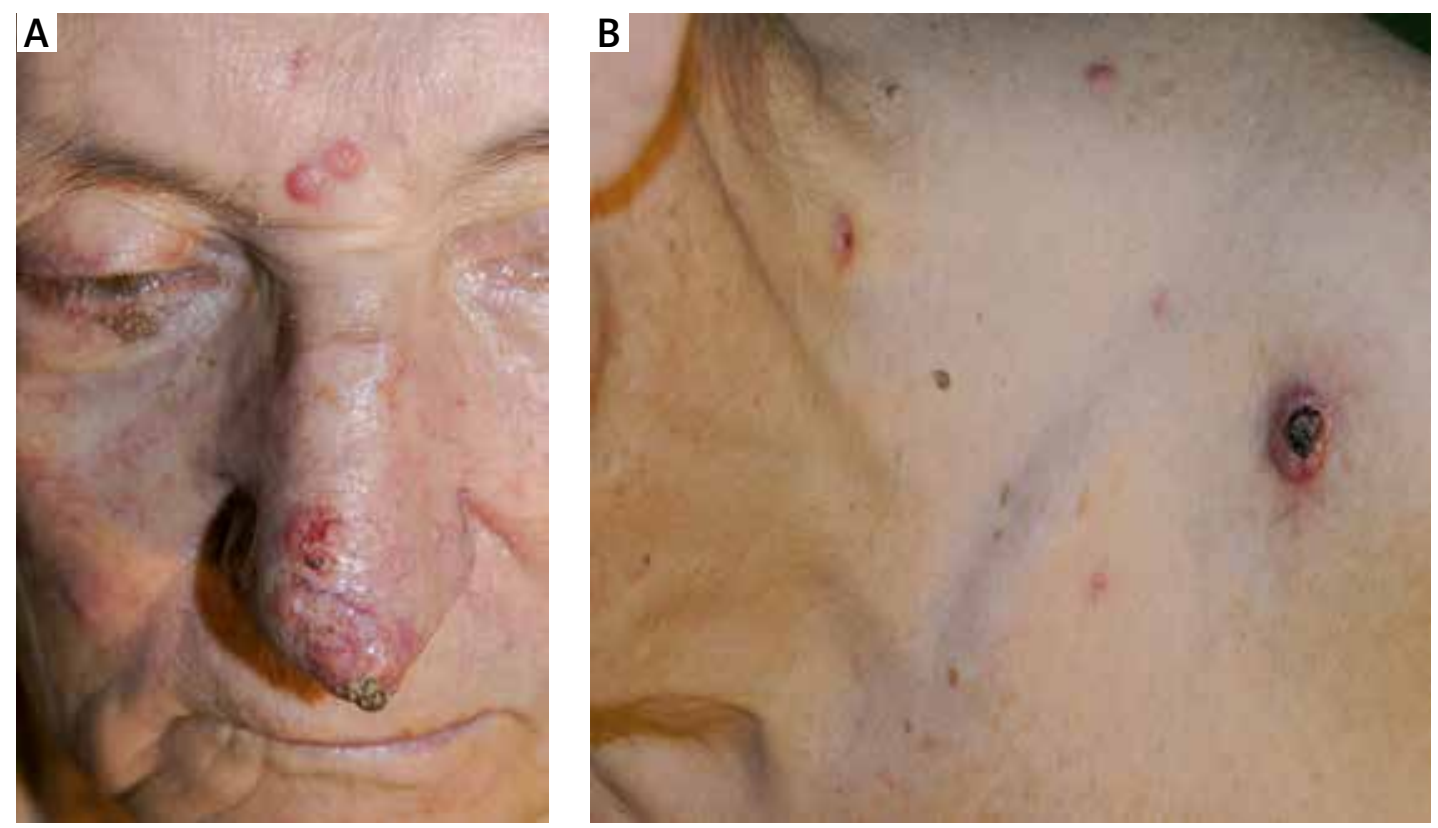

Figure 1. Keratoacanthoma-like metastatic lesions on the nose and forehead (A) and on the shoulder (B)

Address for correspondence: Alina Jankowska-Konsur MD, PhD, Department of Dermatology, Venereology and Allergology, Wroclaw Medical University, 1 Chałubińskiego St, 50-368 Wroclaw, Poland, phone: +48 691033 632, e-mail: alina.jankowska-konsur@umed.wroc.pl Received: 2.06.2017, accepted: 12.07.2017. 

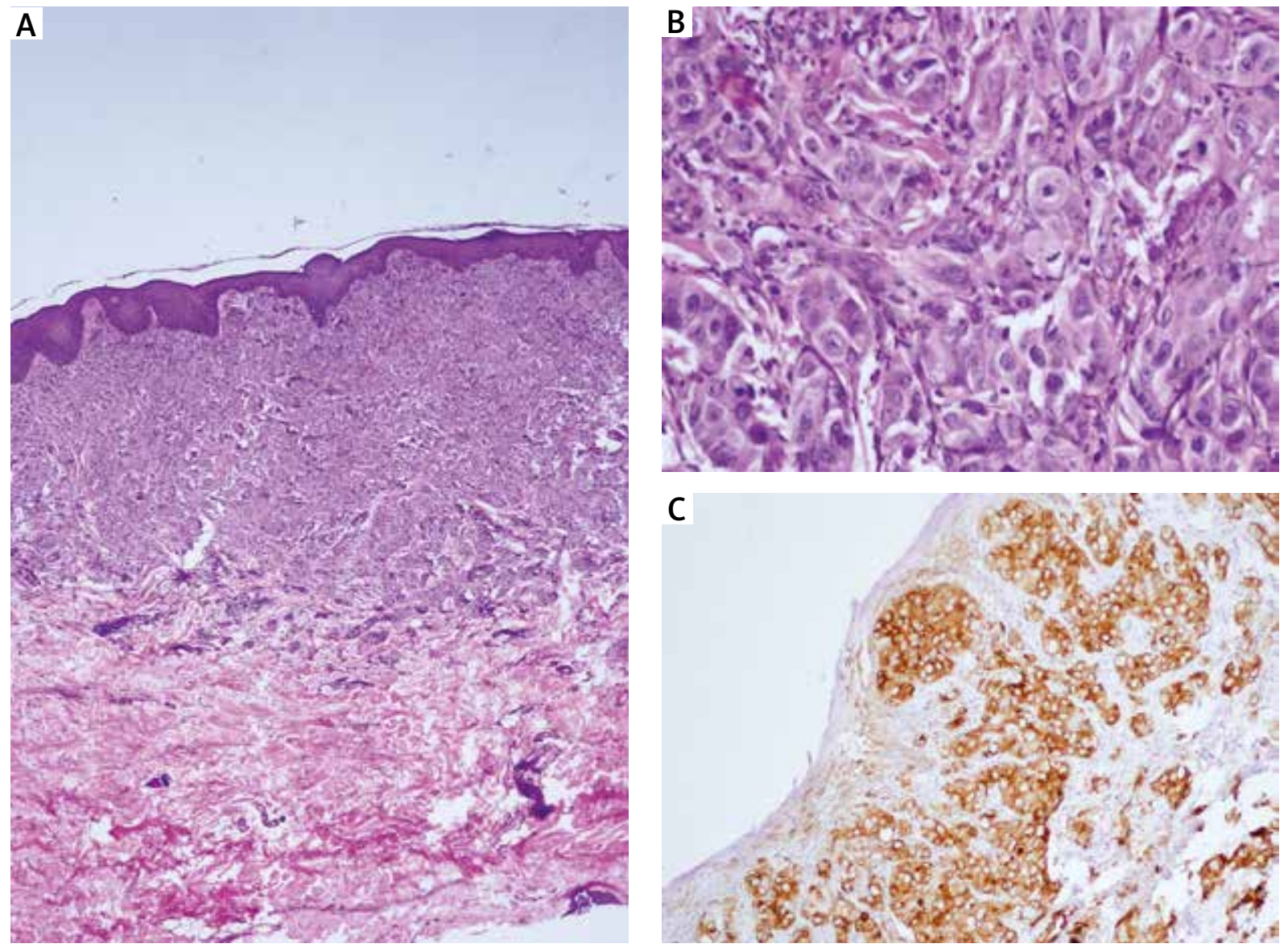

Figure 2. A - metastatic carcinoma: the cicumscribed nodular growth pattern. $\mathrm{H}+\mathrm{E}$, magn. 20x; $\mathbf{B}$ - the features of poorly differentiated carcinoma. $\mathrm{H}+\mathrm{E}$, magn. 200x; C - metastatic carcinoma: the tumor cells strongly positive for EMA, magn. $100 x$

lesions developed rapidly during the 2-month period preceding hospitalization.

The patient had a history of prostate cancer (Gleason score $3+3$ ) treated with radiotherapy and hormonal therapy 4 years ago, hypertension and emphysema. He also suffered from liver cirrhosis secondary to viral hepatitis B, followed by hepatocellular carcinoma treated with transcatheter arterial chemoembolization (TACE) a year ago.

On admission, laboratory tests revealed leukopenia $\left(2370 / \mathrm{mm}^{3}\right)$, anemia $\left(\right.$ RBC $4.10 \times 10^{6} / \mu \mathrm{l}$, HGB $\left.13.0 \mathrm{~g} / \mathrm{dl}\right)$, thrombocytopenia $(49000 / \mu \mathrm{l})$, elevated aspartate aminotransferase (AST) and $\gamma$-glutamyl transferase (GGT) serum levels (78 U/l and $65 \mathrm{U} / \mathrm{l}$, respectively, and D-dimers $(6.65 \mathrm{\mu g} / \mathrm{ml})$. Moreover ultrasound examination of the abdomen showed multiple hyperechogenic lesions covering the liver and $\mathrm{X}$-ray of the thorax showed disseminated round shadows in both middle and lower lung fields.

Biopsy of the skin lesion obtained from the forehead revealed metastatic poorly differentiated carcinoma of unknown origin (Figure 2). The immunochemistry staining showed positive reaction for epithelial membrane antigen (EMA) however other markers (pan-cytokeratin, S-100, Melan A, PSA, AMCAR and CEA) were negative. The expression of Ki67 antigen was lower than 10. Mitotic activity was assessed as 2-3 mitoses per HPF (high power field).

On the computed tomography (CT) scan, new liver tumors were observed and the patient was referred to the Angiosurgery Department to have TACE. During the subsequent therapeutic procedures, the patient's condition deteriorated significantly and he died from the liver cirrhosis decompensation 3 months after the first hospitalization.

Cutaneous metastases from the internal organs clinically mimicking keratoacanthomas are extremely rarely seen in the daily practice. So far, only 11 such cases have been described in the literature [4-6]. In the reported cases, typically, the lesions presented as rapidly growing, solitary, non-tender, firm, dome-shaped nodules with a central plug of keratin, located predominantly on the head (scalp, upper and lower lips, chin). The most common primary malignancy was lung cancer (3 cases), 
followed by laryngeal, breast, esophagus and bronchial cancer, mesothelioma, chondrosarcoma, anaplastic large cell lymphoma and malignant melanoma (single cases) [6]. In the literature there have been only three descriptions of multiple keratoacanthoma-like metastases. They were first symptom of the underlying lung cancer in the first case [7]. In the second case, the metastases of the laryngeal cancer were observed in the location of the patient's radiation port [8]. Multiple lesions resembling generalized eruptive keratoacanthomas of Grzybowsky have been described in one case only (a patient with malignant melanoma) $[9,10]$.

Keratoacanthoma-like metastases were typically observed in middle-aged and elderly patients (age range: 53-79 years). They tended to appear in the course of previously diagnosed internal malignancy rather than they were the first sign of the disease ( 2 cases). The appearance of the lesions was a bad prognostic factor for the majority of the patients and correlated with short survival and disease progression.

In the presented case, the typical keratoacanthomalike lesions were disseminated in three body regions (head, neck and trunk) and were a sign of the advanced malignancy. Unfortunately, the performed diagnostic procedures and rapidly worsening patient's condition did not allow to establish the primary site of malignancy. An important conclusion of the described case is the need to perform a histopathological examination in case of skin lesions resembling keratoacanthoma in a patient with a history of cancer.

\section{Conflict of interest}

The authors declare no conflict of interest.

\section{References}

1. Reingold IM. Cutaneous metastases from internal carcinoma. Cancer 1966; 19: 162-8.

2. Briasoulis E, Pavlidis N, Felip E; ESMO Guidelines Working Group. Cancers of unknown primary site: ESMO clinical recommendation for diagnosis, treatment and follow-up. Ann Oncol 2008; 19 Suppl 2: 106-7.

3. van de Wouw AJ, Jansen RL, Speel EJ, Hillen HF. The unknown biology of the unknown primary tumour: a literature review. Ann Oncol 2003; 14: 191-6.

4. Reich A, Kobierzycka M, Woźniak Z, et al. Keratoacanthomalike cutaneous metastasis of lung cancer: a learning point. Acta Derm Venereol 2006; 86: 459-60.

5. Riahi RR, Cohen PR. Clinical manifestations of cutaneous metastases: a review with special emphasis on cutaneous metastases mimicking keratoacanthoma. Am J Clin Dermatol 2012; 13: 103-12.

6. Lin JH, Lee JY. Primary cutaneous CD30 anaplastic large cell lymphoma with keratoacanthoma-like pseudocarcinomatous hyperplasia and marked eosinophilia and neutrophilia. J Cutan Pathol 2004; 31: 458-61.
7. Sreedevan V, Nair SP, Kumar GN, et al. Keratoacanthoma like secondaries on the scalp. Indian I Dermatol Venereol Leprol 2009; 75: 523-4.

8. Ellis DL, Riahi RR, Murina AT, Cohen PR. Metastatic laryngeal carcinoma mimicking eruptive keratoacanthomas: report of keratoacanthoma-like cutaneous metastases in a radiation port. Dermatol Online J 2014; 20: pii: 13030/qt3s43b81f.

9. Anzalone CL, Cohen PR. Generalized eruptive keratoacanthomas of Grzybowski. Int J Dermatol 2014; 53: 131-6.

10. Reed KB, Cook-Norris RH, Brewer JD. The cutaneous manifestations of metastatic malignant melanoma. Int J Dermatol 2012; 51: 243-9. 